\title{
Overnight orthokeratology: the treatment and control of childhood myopia comes of age
}

Overnight orthokeratology is a refinement or advancement of the clinical technique of contact lens-based orthokeratology
(OK), which has been in use

(OK), which has been in use
worldwide since the 1960s. It is worldwide since the 1960 s. It is
a corneal refractive therapy used in the treatment of conditions of the eye, principally myopia (short-sightedness), but also presbyopia (long-sightedness), hyperopia (age-related loss of ability to focus on near objects) and astigmatism (an imperfection in the curvature of the cornea). Dr César Villa Collar and his team in the Wisin Research Group at the is at the forefront of researh is at the forefront of research orthokeratology technique for myopia control in children.

hildhood and adolescent myopia
(short-sightedness) has increased substantially in recent decades, reaching a prevalence of up to $25 \%$ in the West and $80 \%$ in East Asia, where it has been called an epidemic. Myopia is characterised by blurring of objects viewed at a distance and is commonly eyeball This change in shape causes the refractive ima formed by the corne and the lens to fall in front of instead of onto, the photoreceptors of the retin.

In children who do not develop myopia, the axial length (AL) of the eye increases rapidly at young ages, then slows and stabilises. It is believed that in those who do develop myopia, continued axial elongation is the primary component in myopia progression.

Myopia is measured in diopters (D) where a greater negative number indicates greater severity, and high levels (-6.00D and above) are associated

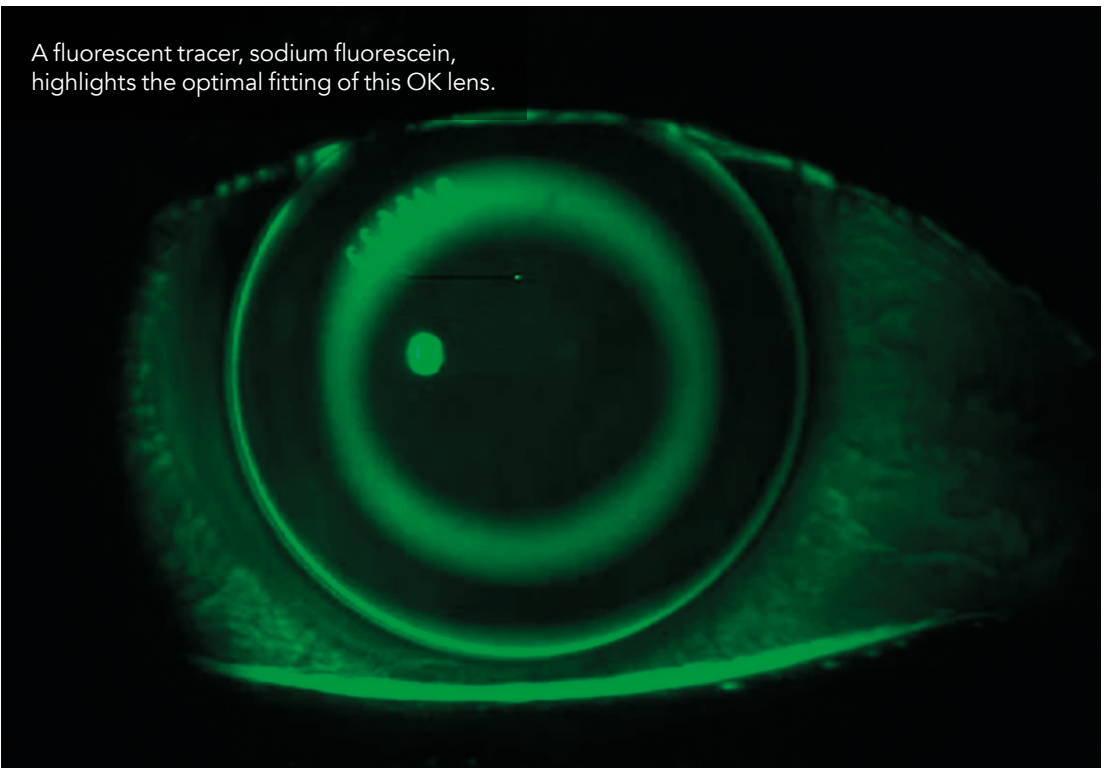

the eye including retinal detachment and glaucoma, and therefore increased healthcare costs.

Efforts to counter this have prompted renewed research into methods of correcting the refractive error but also progression in children.

A BRIEF HISTORY

George Jessen was the first in the world to pioneer and use the technique he called "orthofocus" to correct refractive errors with rigid corneal contact lenses. In 1971 orthokeratology was defined by the International Orthokeratology Section of the National Eye Research Foundation as "the reduction, modification or elimination of refractive anomalies by the programmed application of contact lenses". Since then there have been significant changes in OK lens design materials and wearing schedules Pefinition remains valid.

The first OK lenses could only be worn during the day, as they were made from polymethyl methacrylate (PMMA), mpermeable to oxygen. This aspect bave PMMA lenses a tendency to cause hypoxia (oxygen deficiency) even during open-eye wear, so overnight wear was not possible. In the 1980s, rigid gas-permeable lenses became available. Their high oxygen permeability allowed them to be used successfully in extended daily wear and overnight wear, heralding the advent of overnight $\mathrm{OK}$, in which the lenses are wo only during sleep and removed shortly after waking. The corrective effect of the lenses then provides the patient with clear unaided vision throughout the day. During the course of the day there is some revers

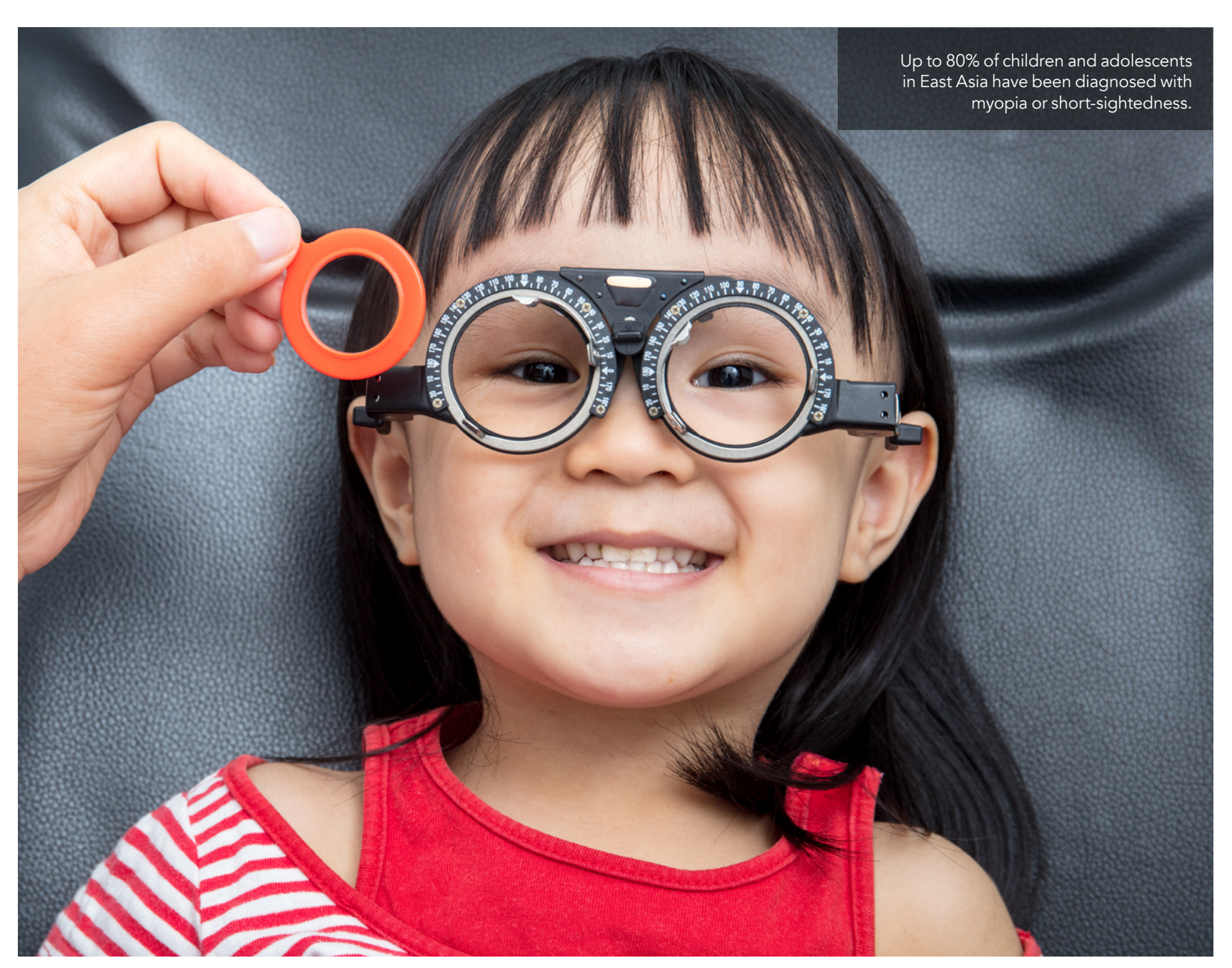

Orthokeratology contact lens wear reduces axial elongation in comparison to distance single-vision spectacles in children MECHANISM OF ACTION The beneficial effect of overnight OK was first achieved by using fitted rigid contact (specifically flattening and thinning of the uppermost cell layer, the epithelium, in the (a) modify or eliminate refractive error.

\begin{tabular}{|c|c|c|c|c|c|c|}
\hline REGION & 2000 & 2010 & 2020 & 2030 & 2040 & 2050 \\
\hline Central, East, West and Southern Africa & 4.6 & 6.7 & 9.9 & 14.4 & 20.1 & 26.9 \\
\hline North and Middle East Africa & 14.6 & 23.3 & 30.5 & 38.8 & 46.3 & 52.2 \\
\hline Latin America and the Caribbean & 16.6 & 22.3 & 30.2 & 38.4 & 46.4 & 52.3 \\
\hline East and South East Asia \& Pacific & 39.6 & 45.0 & 47.0 & 55.7 & 60.5 & 64.5 \\
\hline South and Central Asia \& Australasia & 15.1 & 21.5 & 29.6 & 38.2 & 45.8 & 51.8 \\
\hline Europe & 20.1 & 26.8 & 34.5 & 41.7 & 48.6 & 53.5 \\
\hline North America & 28.3 & 34.5 & 42.1 & 48.5 & 54.0 & 58.4 \\
\hline Oceania & 5.0 & 6.7 & 9.1 & 12.5 & 17.4 & 23.8 \\
\hline GLOBAL & 22.9 & 28.3 & 33.9 & 39.9 & 45.2 & 49.8 \\
\hline
\end{tabular}

Modified from Holden BA et tal. Ophthalmology 2016; 123(5): 1036-1042 https://doi.org/10.1016/1/
ohhtha. 2016.01.006 originally published under CC BY-NC-ND 4.0.

Renewed interest in the use of overnight OK has largely been due to the development of what are called "reverse-geometry" lenses. Rather "han having the conventional concave reverse-geometry lenses are structured as a centrat optic zone fitted flat relative to the central cornea and surrounded by one or more steeper secondery or "reverse" curves which act to stabilise the lens. This central treatment zone encompasses the patient's pupil diameter.

Reverse-geometry lenses came into accepted and widespread use in the 1990s and have undergone successive refinements to reach the point where they are widely used around the world for the temporary correction of low to moderate myopia (up to about -4.00D) although there are reports of its use for Asia, which may be a reflection of the Asia, which may be a retection of the of myopia in this region. 
MYOPIA CONTROL

Recent studies have uncovered an

of myopia. This is what is known as

myopia control and it is thought to stem reduction in the stimulus for eye growth.

It has been estimated that $80 \%$ of overnight OK lens wearers in East Asia the progression of myopia during the
critical stages of myopia development

Dr César Villa Collar has a long and refractive errors). He and his team at the Universidad Europea de Madrid have conducted numerous studies specifically related to myopia control. They have

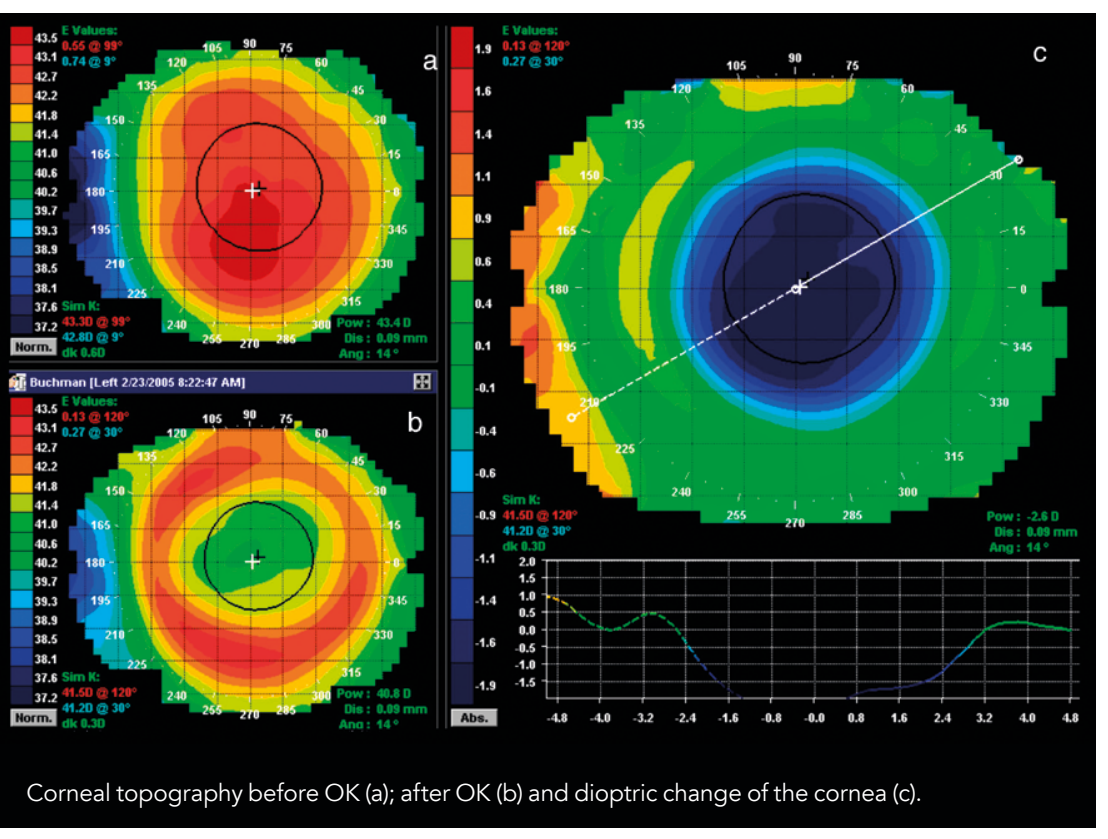

slowing down or halting the progression

directly from the optical configuration

and design of modern reverse geometry lenses and how they affect, in this case, not the central cornea but the periphery where they are thought to bring about a are 18 years or under and are using it specif cally for the pupose of countering

It is this perceived ability to slow or control which is at the centre of the research drive and strategy of fitting overnight OK lenses at much younger ages than previously. distinguished research pedigree founded upon years of work in orthokeratology, corneal topography (mapping the shape and contours of the cornea) and ocular aberrometry (measuring the degree of found that overnight OK contact lens wear

myopia in comparison to distance single- vision spectacles in children (2012). They also discovered a trend toward a reduction in the rate of axial elongation (2017): following seven years of lens wear, those using overnight $\mathrm{OK}$ compared to single

\section{SAFETY OF OVERNIGHT} ORTHOKERATOLOGY

The increasing use of overnight $O K$ and

$80 \%$ of overnight orthokeratology lens wearers in East Asia are 18 years or under and are using it specifically for the purpose of countering myopic progression. concerns about safety.Specifically, there was a worry that the treatment might lead to an increased risk of eye infection (microbial keratitis) compared to open-eye or daily procedure has been shown consistently to be safe. Almost all the cases of microbia keratitis reported in an extensive 2005 review article came from patients of the practice is now closely controlled.

BEYOND MYOPIA

The use of overnight OK for treatment of mild to moderate myopia and myopia he relative imporance of the researches into it, in terms of volume of academic output.

They found that Dr Villa Collar ranks third on a worldwide list of the most productive authors on the subject. of publications on orthokeratology was in the period 2003 to 2007. The largest number of documents produced on the subject, accounting for nearly $40 \%$ of the total output, was between 2013 of interest in research in this field.

This increase in research activity and interest coincides with the validation of reliable results in myopia control in childhood and adolescence using overnight orthokeratology, and is expected to grow with its increasing acceptance, and as its application as an effective method of controling myopia progression becomes more with some je. fication, with some justification, that overnigh has come of age. in the rate of as experienced a reduction

better understanding of the mechanism

of corneal reshaping, particularly in relation wear lenses. These concerns have turned out to be largely theoretical. In the West the unregulated practitioners in East Asia wher

control is now well-documented. and in lens design have the technique successful treatment of high myop in p to -12.00D. Further refinements, called toric designs, with modifications in the optical or the peripheral zone of he lens, have facilitated the treatmen hyperopia up to 3 diopters and

\section{LOOKING AHEAD} Perhaps one of the best indicators of people are talking about something. Wrive a good illustration of this it is produced by DrVilla Collar and his colleagues and published in 2018. This was a "bibliometric study" of scientific esearch on overnight $\mathrm{OK}$, in other words

\section{Behind the Research}

\section{Dr César Villa Collar}

E: Cesarvilla@universidadeuropea.es W: https://www.researchgate.net/profile/Cesar_Villa_Collar W: https://scholar.google.es/citations?user=SbeM0_0AAAAJ\&hl=es\&oi=ao

\section{Research Objectives}

Dr César Villa Collar employs an overnight orthokeratology control in children.

\section{Detail}

Dr César Villa Collar

Calle Tajo s/n. 28670 Villaviciosa de Odón

Madrid

Bio

DrVilla Collar gained his doctorate in Optics, Optometry and Vision from the Complutense University of Madrid. He is the Managing Editor of the Journal of Optometry and a Fellow of the American Academy of Optometry and the European Academy of Optometry and Optics. He is 列 ch Group of this university.

Collaborator

Vision Research Group

Cristina Alvarez Peregrina, PhD

- Alicia Ruiz Pomeda, PhD

\section{GIV \\ ue Universidad}

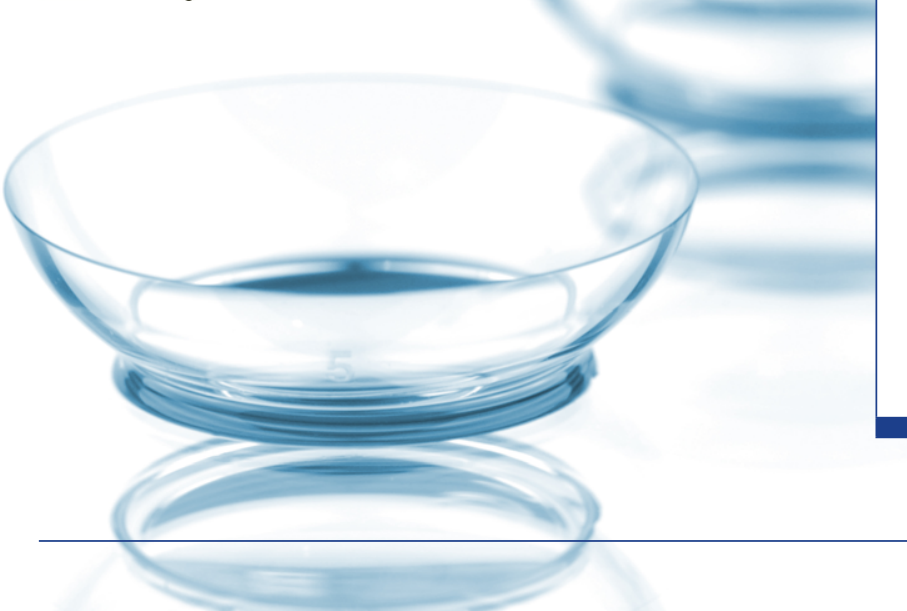

\section{References}

- Carr B, Stell W (2017). The science behind myopia. NCBI. Available at: https://www.ncbi.nlm.nih.gov/books/ NBK470669/Accessed 18 July 2019

- Hou W, Norton T, Hyman L, Gwiazda J (2018). Axial
Elongation in Myopic Children and its Association W Myopia Progression in the Correction of Myopia Evaluation
Trial. Eye \& Contact Lens: Science \& Clinical Practice; 44(4): Trial. Ey $248-259$

- Jessen G. Orthofocus techniques. 1962 Contacto; 6:200 - 204 - Li S, Kang M, Wu S, Liu L, Li H, Chen Z, Wang N. (2016) slowing axial elongation in myopic children by meta-analysis. Curr Eye Res; $41(5): 600-8$

- Santodomingo-Rubido J, Villa-Collar C, Gilmartin B, GutierrezOrtega R (2012) Myopia control with orthokeratology contact lenses in Spain: refractive and biometric changes. Invest (8).5060-5 - Santodomingo-Rubido J, Villa-Collar C, Gilmartin B,
Gutierrez-Ortega R, Sugimoto K. (2017). Long term efficacy of of childhood myopia. Curr Eye Res; 42(5): $713-720$

- Swarbrick H. (2006). Orthokeratology review and update. Clin

- Villa-Collar C, Alvarez-Peregrina C, Hidalgo Santa Cruz F, Research on Overnight Orthokeratology Eye \& Contact Lific Research on Overnight
Science \& Clinical Practice. 44(5):344-349

- Watt K, Swarbrick H. (2005). Microbial keratitis in overnight orthokeratology: review of the first 50 cases. Eye Contact

\section{Personal Response}

The interest in overnight orthokeratology from the increase in recent years. With modern materials and technology to hand where would you anticipate or like the next major development in the field to be? II In order to control myopia, an important future development will be customised treatment to improve moulding that does not alter the aspherical curvature of the should mould the cornea to get a multifocal shape that compensates for presbyopia. Finally, it would be interestin to increase the lifespan of the lenses - at present, hey must be replaced once a year. 